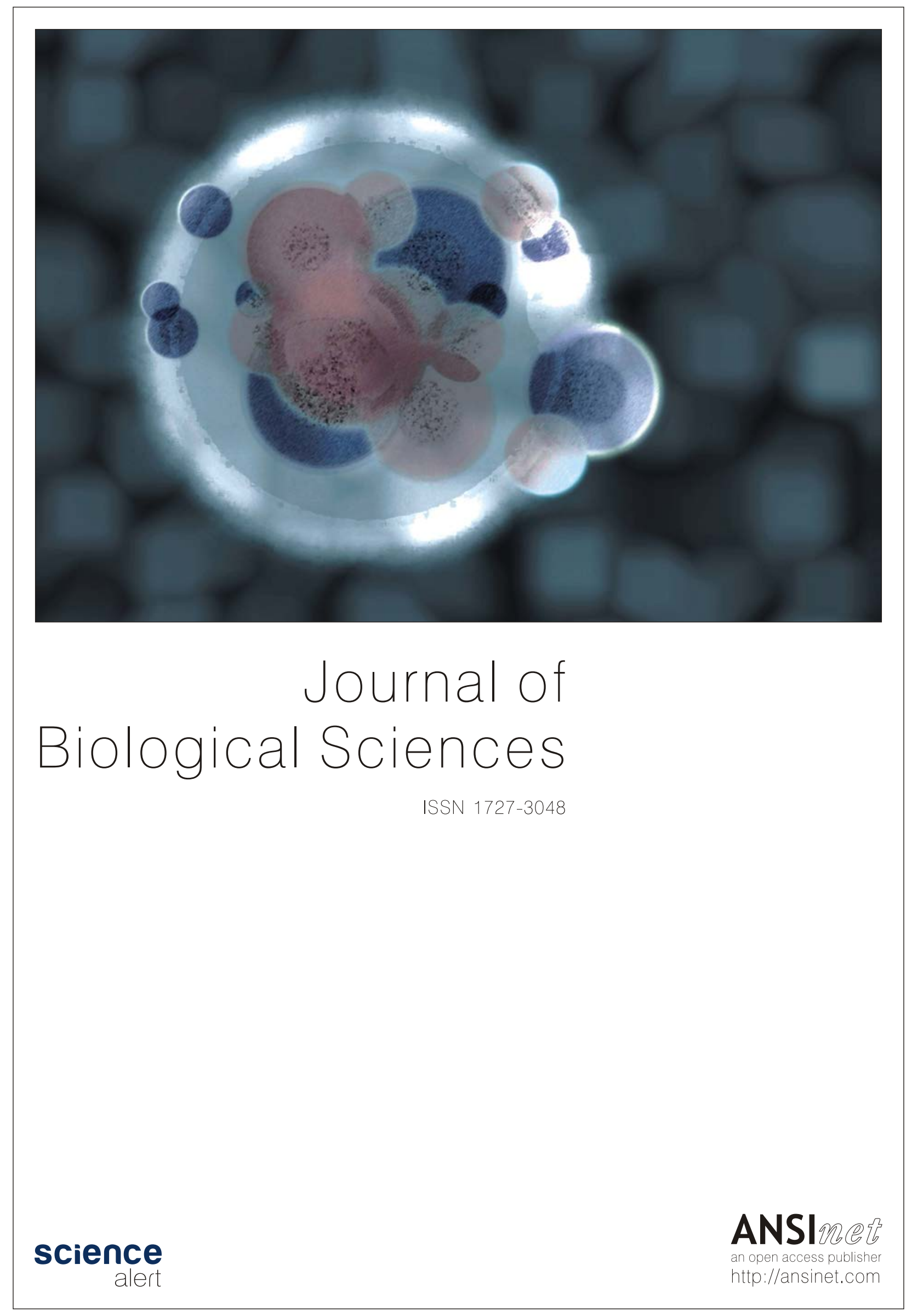




\title{
Formulation and Evaluation of an Adaptogenic Formula to Improve Performance of Athletes Practicing Anaerobic Exercises
}

\author{
${ }^{1}$ Fawzi Amin El-Shobaki, ${ }^{1}$ Hany Mohamed Ahmed Wahba, ${ }^{1}$ Maha Hanafy Mahmoud, ${ }^{1}$ Yusr Ibrahim Kazem, \\ ${ }^{1}$ Ibrahim Hassan Badawy and ${ }^{2}$ Ahmed Said Zahran
}

${ }^{1}$ Department of Nutrition and Food Science, National Research Centre, 33 EL Bohouth St., P.O. 12622, Dokki, Giza, Egypt

${ }^{2}$ Department of Individual Training, Faculty of Physical Education, Helwan University, Cairo, Egypt

\section{Abstract}

Background and Objectives: The increased physical performance, delayed muscular fatigue, reduced recovery time and improved endurance is the ultimate aim of mostly all athletes. An adaptogenic formula was prepared for athletes of anaerobic exercises for achieving these goals. Materials and Methods: Fifteen healthy male taekwondo athletes of age $20.29 \pm 0.81$ years participated in this program. The formula is composed of whey protein concentrate, L-arginine, root extract of Korean red Panax ginseng and cocoa powder. Participants continued on the program of the adaptogenic formula for 4 weeks. Anthropometric measurements, medical and mood states assessment and physical performance were evaluated. Biochemical evaluation including serum lactate dehydrogenase-5 (LDH-5), creatine kinase (CK), cortisol, nitric oxide (NO), malondialdehyde (MDA), total antioxidant capacity (TAC), complete blood count (CBC), liver and kidney functions were followed before and after enrolling in the program. Results: There was a highly significant improvement in recovery time and physical performance that reflects the efficiency of the formula to remove or combat undesirable metabolic by-products of the anaerobic oxidation. Biochemical evaluation revealed a significant reduction in LDH-5, CK, cortisol, a non-significant decrease in MDA, a significant increase in $\mathrm{NO}$ and a non-significant rise in $\mathrm{TAC}$, while there was no change in $\mathrm{CBC}$, liver and kidney functions indicating that the prepared formula was safe and could direct the metabolism towards clearance of by-products due to anaerobic oxidation that cause fatigue and cell degradation. Conclusion: It can be concluded that using this formula by athletes of anaerobic exercises leads to getting rid of anaerobic by-products thus helps to combat muscular fatigue, enhance recovery time and improve athletic performance provided that it can be used for longer time.

Key words: Adaptogenic formula, anaerobic exercise, muscular fatigue, LDH-5, CK, cortisol

Received: March 18, 2018

Accepted: May 16, 2018

Published: June 15, 2018

Citation: Fawzi Amin El-Shobaki, Hany Mohamed Ahmed Wahba, Maha Hanafy Mahmoud, Yusr Ibrahim Kazem, Ibrahim Hassan Badawy and Ahmed Said Zahran, 2018. Formulation and evaluation of an adaptogenic formula to improve performance of athletes practicing anaerobic exercises. J. Biol. Sci., 18: $231-242$.

Corresponding Author: Maha Hanafy Mahmoud, Department of Nutrition and Food Science, National Research Centre, Dokki, Giza, Egypt Tel: $+201225757319,+201064568707$

Copyright: @ 2018 Fawzi Amin El-Shobaki et al. This is an open access article distributed under the terms of the creative commons attribution License, which permits unrestricted use, distribution and reproduction in any medium, provided the original author and source are credited.

Competing Interest: The authors have declared that no competing interest exists. 


\section{INTRODUCTION}

Taekwondo is an example for anaerobic sports. It is the art of kicking and punching and it is the most popular marital art worldwide that is practiced by about million participants daily and studied in more than 140 countries $^{1}$. Among the main factors which determine the performance of the players is the anaerobic capacity for rapid and explosive movements ${ }^{2}$.

Low athletic performance is mainly due to feeling fatigue and prolonged recovery time ${ }^{2}$. In general, exercises may be classified into aerobic and anaerobic according to the energy system they utilize based on intensity and duration of the exercise. These energy systems include the phosphogenic or anaerobic ATP-PC system with a very short duration up to $10 \mathrm{sec}$ and high intensity including explosive movements. Another anaerobic system is the glycolytic anaerobic system associated with lactic acid production which occurs at short duration up to $2 \mathrm{~min}$ and moderate intensity. The third system is the aerobic or oxidative system with a long duration and low intensity ${ }^{3}$. During high peak metabolic power, the body of athletes relies mainly on anaerobic metabolism, the phosphogen ATP-PC and the anaerobic glycolysis which result in the formation of undesirable end-products mainly inorganic phosphate and lactate, respectively. These metabolites exert a limiting effect on muscle function causing fatigue that results in reduced performance ${ }^{4-6}$. Actually, the body doesn't rely exclusively on one energy system, but there is a coordination between the three energy systems to varying degrees depending on the intensity and duration of the exercise ${ }^{7}$.

The interference to improve performance can be either to increase oxygen supply during exercise or to increase efficiency of energy utilization. Another rout is to minimize the metabolic by-products of anaerobic oxidation. Many chemicals, food components and dietary supplements that can perform this role were discovered before and were used by Russian Scientists 8 . They called these compounds, adaptogens. Generally, adaptogens are metabolic regulators that can increase the resistance of the body to harmful stressors of physical, biological and chemical natures ${ }^{9}$. Adaptogens were assumed to reduce damage due to stress, increase working capacity and mental performance and can exert normalizing effect on pathological state. Adequate adaptogens are important for optimizing exercise performance and combating fatigue ${ }^{10}$.

There are several plant sources that contain compounds able to perform the adaptogenic roles. Root extract of Korean red Panax ginseng (C.A. Meyer) has been well known for enhancing human performance either mental or physical ${ }^{10}$, due to energy restoring ${ }^{11}$, anti-fatigue or to reduce muscle damage induced by intensive exercise ${ }^{12}$. It also possesses an antioxidativepower ${ }^{13,14}$.

L-arginine supplementation was found to enhance strength and help muscle recovery either in aerobic or anaerobic exercises ${ }^{15}$. It was found to reduce $\mathrm{O}_{2}$ cost of the exercise of moderate intensity and to enhance the tolerance of severe-intensity exercise ${ }^{16}$. This is done through the signaling molecule nitric oxide (NO) which is synthesized from $\mathrm{L}$-arginine by the action of the enzyme endothelial NO synthase. L-arginine has been used by athletes to increase strength and to elevate lean muscle mass ${ }^{17}$.

Athletes of high intensity training have more dietary protein needs reached up to two times the Daily Recommended Allowance ${ }^{18}$. Whey protein is a good source for essential amino acids and branched chain amino acids which are very important for tissue maintenance during exercise, for preventing catabolic actions, for decreasing protein degradation and enhancing protein synthesis ${ }^{18,19}$. It was reported that whey protein supplementation in young resistance trained men and women resulted in improving exercise performance ${ }^{20}$.

In studies looking for the effect of adaptogenic compounds that help athletes to perform better and delay the feeling of fatigue, it is needed to have good reliable biochemical parameters for muscle fatigue assessment. The most reliable biochemical parameters for assessment of muscular fatigue are the two enzymes LDH-5 (lactate dehydrogenase, isoenzyme- 5 which predominates in muscles) and CK (creatine kinase) which are the enzymes responsible for removal of the by-products of muscular fatigue $^{21}$. Also, cortisol which is a glucocorticoid hormone secreted by the adrenal gland rises in the circulation due to muscular damage as a result of heavy physical activity ${ }^{22}$. In addition, nitric oxide (NO) which is a compound of regulatory effect in skeletal muscles helps for muscular repair and rebuilding ${ }^{15}$.

The main objective of this study is to evaluate the effect of the composed adaptogenic formula formed from L-arginine, whey protein concentrate, ginseng and cocoa powder on the physical and metabolic changes that occur to athletes during performance of intensive exercises where energy supply depends mainly on anaerobic oxidation such as taekwondo players. Also, to detect the improvement by following the physical performance and biochemical parameters that reflect the removal of the by-products of anaerobic oxidation so as to enable the formula to be used together with those that already exist in the market. 


\section{MATERIALS AND METHODS}

Subjects: Fifteen male athletes from Taekwondo Sport Team were included in this study. Their mean age was $20.29 \pm 0.81$ years. Taekwondo Sport was chosen as an example for the domination of anaerobic exercises. All subjects were healthy normal persons with no history of chronic illness. The exclusion criteria included individuals with any disease, those with anabolic steroids consumption background and those who received any other dietary supplement. The participants were informed about the objectives of the study and their permission in the form of written consent was obtained. The study protocol was done in accordance with the guidelines stated by the Ethical Committee of the National Research Centre and it was approved by the committee. This study was started in September, 2016 till February, 2017.

Materials: The formula given to the athletes is composed of whey protein, L-arginine, Panax ginseng C A Meyer and cocoa powder. Soluble whey protein was obtained from Imtenan Health Shop, lot No. 10, The Industrial Area, Obour City, (Egypt), L-Arginine Hydrochloride from SAGA Sciences Inc., Vancouver, BC, (USA), ginseng from Yanbian, Bitoshan Ltd., (Korea) and cocoa powder from local market.

\section{Methods}

Composition of the formula: Before composing the formula, the protein content of whey concentrate was determined according to AOAC official methods of analysis ${ }^{23}$. It was found to be $47.25 \pm 1.22 \mathrm{~g}$ protein $/ 100 \mathrm{~g}$ whey protein concentrate.

The amino acid content was determined according to the procedure described in $\mathrm{AOAC}^{24}$ using the amino acid analyzer (Beckman, USA) (Table 1).

Based on the arginine content of the whey as shown in Table 1, the $40 \mathrm{~g}$ whey protein concentrate added to the formula contains $2.24 \mathrm{~g} \mathrm{~L}$-arginine. Two $\mathrm{g}$ of L-arginine was added to reach a concentration $4.24 \mathrm{~g}$ of L-arginine as modified from Bailey et al.16. The amount of ginseng added was an average amount used by other authors and depends on the concentration of the active ingredient "ginsenosides" present in ginseng, which amounted to $5 \mathrm{mg} /$ each gram of the Korean ginseng. Cocoa powder was added as a flavor. Thus, the composed adaptogenic formula included $40 \mathrm{~g}$ of whey protein concentrate, $2 \mathrm{~g}$ of L-arginine, $9 \mathrm{~g}$ of Korean red ginseng root extract and $9 \mathrm{~g}$ of cocoa powder. All these constituents were mixed well together and the resultant powder was given in special bags each containing $60 \mathrm{~g}$ once daily. The contents of each bag was dissolved in a cup of skimmed milk immediately before being used. The study lasted for 4 weeks.

The formula was subjected before use to organoleptic evaluation. Fifteen panelists were each given the formula and asked to give score out of 20 for different organoleptic characteristics, such as color, taste, odor, appearance and overall acceptability of the supplement. Data obtained revealed good taste and appearance and very good acceptability.

Biological evaluation: The following investigations were done before and after the end of the period of the study which lasted for 4 weeks:

- Full medical history: Including history of any chronic illness, operations, medications and family history

- Full clinical examination: Which including blood pressure, resting heart rate (pulse), skin, tongue, mouth discoloration or ulcers, heart, chest, abdomen examination

- Mood states assessment and general fitness evaluation: Including, mood, memory, focus during training, enjoying training, , recovery time after training, time before feeling fatigue, feeling sleepy and sleep quality

- Anthropometric measurements: That included weight and height, then body mass index was calculated as, weight in $\mathrm{kg} /$ height in $\mathrm{m}^{2}$. Body fat as a percent from body weight and lean body mass (LBM) was measured according to Spungen et al.25

- Physical performance

- Biochemical analysis

Table 1: Amino acid profile of whey protein concentrate

\begin{tabular}{lclc}
\hline Amino acid & Concentration (\%) & Amino acid & Concentration (\%) \\
\hline Aspartic (ASP) & 3.82 & Tyrosine (TYR) & 2.00 \\
Therionine (THR) & 1.78 & Phenylalanine (PHE) & 2.56 \\
Serine (SER) & 1.95 & Histidine (HIS) & 1.21 \\
Glutamic (GLU) & 9.13 & Lysine (LYS) & 3.05 \\
Glycine (GLY) & 1.10 & Arginine (ARG) & 5.63 \\
Alanine (ALA) & 1.75 & Proline (PRO) & 3.28 \\
Valine (VAL) & 2.90 & Cystine (CYS) & 0.85 \\
Isoleucine (ILE) & 2.13 & Methionine & 1.21 \\
Leucine (LEU) & 3.85 & - & - \\
\hline
\end{tabular}




\section{General physical fitness evaluation and specific assessment} for the anaerobic power: For general physical evaluation, a questioner for subjective physical fitness and general well being was applied. Each participant was interviewed as one to one and the evaluation was put on 5 points scale, where $1=$ least and 5 maximum.

The questioner included the following items:

- Time spent before feeling fatigue: $1=30 \mathrm{~min}, 2=50 \mathrm{~min}$, $3=70 \mathrm{~min}, 4=90 \mathrm{~min}$ and $5=110 \mathrm{~min}$

- Recovery time (time spent before being able to perform another training session): $1=$ Can play after $2 \mathrm{~h}, 2=$ Can play after 2-3 h, 3= Can play after 4-6 h, $4=$ Can play after 6-8 $\mathrm{h}$ and $5=$ Cannot play on the same day

- Sleep duration: $1=3-4 \mathrm{~h}, 2=4-5 \mathrm{~h}, 3=5-6 \mathrm{~h}, 4=6-7 \mathrm{~h}$ and $5=7-9 \mathrm{~h}$

An assessment for the anaerobic power was carried out before and after enrolling in the program. Each of the following was evaluated for each participant as follows:

- Muscle endurance and performance endurance: The number of each of setups and pushups for one min and the exercise of front turning kick for one min before and after enrolling in the program were done for each participant to determine the muscle endurance and performance endurance, respectively which measured the anaerobic system for energy production that yields lactic acid

- Motor speed and explosive strength: The exercise of the front turning kick for $15 \mathrm{sec}$ and the exercise of standing long jump before and after enrolling in the program were done for each participant to determine the motor speed and explosive strength, respectively which measured the ATP-PC anaerobic energy system

Biochemical analysis: A blood sample was withdrawn from each participant directly after workout at the beginning of the study before the intake of the formula and at the end of the study. The following biochemical parameters were done:

- Parameters for the catabolic status: Human lactate dehydrogenase-5 (LDH5) was assessed by ELISA technique according to manufacturer's instructions with a kit for human having the Cat. No. of 90312 and obtained from Glory Science Co., LTD, USA ${ }^{26}$, using ELISA Reader (ELISA Reader Model Start F, Awareness Technology, Inc., Palm City, FL, USA). Cortisol was determined by ELISA kit according to manufacturer's instructions with a product code of 3625-300 and obtained from Monobind Inc., California 92630, USA ${ }^{27}$. Creatine phosphokinase (CK) was determined colorimetrically by a kinetic method with a kit of Cat. No. of 1001050 obtained from Spinreact ${ }^{28}$, S.A.U., Girona, Spain and the optical density was read on a spectrophotometer (Shimadzu UV-2401 PC, Australia). Nitric oxide (NO) was determined by colorimetric method with a kit of Cat. No. of NO 2533 and obtained from Biodiagnostic Co. ${ }^{29}$, Egypt

- Parameters for oxidative stress: Lipid peroxidation product, malondialdehyde (MDA) was measured as lipid peroxide product by the thiobarbituric acid assay (TBA) according to the method of Draper and Hadley ${ }^{30}$. Total antioxidant capacity (TAC) measured by colorimetric method with a kit of Cat. No. of TA2513 and obtained from Biodiagnostic Co. ${ }^{31}$, Egypt

- Screening for different body functions: Complete blood count (CBC) in whole blood sample was measured by an automated hematology analyzer (Sysmex, XT-1800i, Hamburg, Germany) which uses fluorescence flow cytometry (FFC) technology. The activity of both aspartate amino transferase (AST) and alanine aminotransferase (ALT) (representing the liver function) were determined using a kit obtained from Salucea Co. ${ }^{32}$, Netherlands, with Cat. No. of NS 260001 and NS 264001, respectively. Total protein, urea, creatinine (representing the kidney function) were assessed by colorimetric method with kits obtained from Chronolab systems S. L., Barcelona, Spain with Cat. No. of 101-0240 $33,101-0425^{34}$ and $101-0281^{35}$ for total protein, urea and creatinine, respectively

Statistical analysis: Data were statistically analyzed by the computerized program SPSS software, version "20" for Windows. "Paired-Sample T-Test" was used to compare between mean values of the analyzed parameters before and after intake of the dietary supplement. Data was represented as Mean $\pm S E$. Values were considered significant at $p<0.05$, otherwise were considered non-significant.

\section{RESULTS AND DISCUSSION}

\section{Physical and medical characteristics and profile of mood} states: Data obtained from the clinical sheets showed that the subjects included in this program after the intake of the adaptogenic formula were enjoying relatively better health state than before entering the program. This is evidenced from the measured parameters shown in Table 2 . 
Table 2: Physical and medical characteristics and profile of mood states of all subjects

\begin{tabular}{|c|c|c|c|c|}
\hline \multirow[b]{2}{*}{ Parameters } & \multicolumn{3}{|c|}{ Subjects $(n=15)$} & \multirow[b]{2}{*}{$p$-value } \\
\hline & Before & After & Change (\%) & \\
\hline Age (years) & $20.29 \pm 0.81$ & & & \\
\hline Height $(\mathrm{cm})$ & $174.29 \pm 1.34$ & & & \\
\hline Weight (kg) & $64.14 \pm 1.96$ & $64.43 \pm 1.88$ & 0.45 & NS \\
\hline BMI $\left(\mathrm{kg} \mathrm{m}^{-2}\right)$ & $21.11 \pm 1.85$ & $21.16 \pm 0.57$ & 0.24 & NS \\
\hline Body fat (\%) & $7.85 \pm 1.00$ & $7.78 \pm 0.77$ & -0.89 & NS \\
\hline $\operatorname{LBM}(\mathrm{kg})$ & $58.31 \pm 1.49$ & $58.58 \pm 1.51$ & 0.46 & NS \\
\hline $\mathrm{SBP}(\mathrm{mm} \mathrm{Hg})$ & $110.00 \pm 0.03$ & $110.00 \pm 0.02$ & 0.00 & NS \\
\hline $\mathrm{DBP}(\mathrm{mm} \mathrm{Hg})$ & $80.00 \pm 0.02$ & $80.00 \pm 0.03$ & 0.00 & NS \\
\hline Resting heart rate (pulse) & $75.00 \pm 0.04$ & $76.00 \pm 0.04$ & 0.02 & NS \\
\hline Mood & $4.20 \pm 0.03$ & $4.30 \pm 0.03$ & 2.30 & NS \\
\hline Memory & $4.10 \pm 0.02$ & $4.20 \pm 0.03$ & 4.20 & NS \\
\hline Focus during playing & $3.90 \pm 0.03$ & $4.20 \pm 0.03^{*}$ & 5.10 & 0.000 \\
\hline Enjoying training & $3.90 \pm 0.03$ & $4.60 \pm 0.02^{*}$ & 17.90 & 0.000 \\
\hline Recovery time & $3.00 \pm 0.02$ & $2.00 \pm 0.03^{*}$ & -33.30 & 0.000 \\
\hline Time before feeling fatigue & $3.00 \pm 0.03$ & $5.00 \pm 0.02 *$ & 66.70 & 0.000 \\
\hline Feeling sleepy & $3.00 \pm 0.03$ & $4.00 \pm 0.03^{*}$ & 33.30 & 0.000 \\
\hline Sleep quality & $2.00 \pm 0.02$ & $3.00 \pm 0.03^{*}$ & 50.00 & 0.000 \\
\hline
\end{tabular}

As shown in the table blood pressure (systolic and diastolic) and resting heart rate (pulse), remained stable showing that the adaptogenic formula did not exert any hazard to the heart function or the blood flow. Regarding the physical characteristics or anthropometric measurements (weight, BMI, body fat $\%$ and LBM) of the subjects (Table 2), no statistically significant difference exists between the values measured before entering the program and at the end of intervention. This shows that it exerted no effect on body weight or body fat. Since a decline in cognitive and skill performance has been observed previously after fatiguing exercise ${ }^{36}$, it was important to evaluate the cognitive performance and the mood states of the subjects before and after enrolling in the program. It can be noticed that many of the parameters reflecting the cognition and mood states of the subjects such as mood, memory, focus during playing, enjoyment of training, recovery time after training, time before feeling fatigue, feeling sleepy and sleep quality showed improvement (Table 2). Each of these characters was given a score from 1-5 and were measured twice, once before entering the program and the other at the end. The improvement was significant in case of focusing during playing with a percent change of $+7.7 \%$, enjoying training $(+17.9 \%)$, recovery time after training $(-33.3 \%)$, time before feeling fatigue $(+66 \%)$ and sleep quality $(+50 \%)$. This is again a confirmation that the adaptogen contains ingredients or compounds that help the body to keep fit and even may be more fit than before entering the program (Table 2). Similar results previously reported by other authors agree with these findings. Ginseng products were reported to have a positive effect on the mood, cognitive performance, well-being ${ }^{37}$, as well as anti-fatigue effect ${ }^{38}$. Also, L-arginine supplementation prior to a single bout of eccentric exercise in athletes was reported to alleviate damage of muscle fibers and to preserve its performance capacity of the exercise ${ }^{39}$. Moreover, whey protein supplementation in young resistance trained men and women was reported to improve exercise performance as they enhance muscle protein synthesis ${ }^{20}$. Thus, it seems that the combined and synergistic effect of each of Panax ginseng, $\mathrm{L}$-arginine and whey protein was able to combat fatigue and enhance physical performance. This also proves the importance of including more than one component in the formula.

Exercise performance: Parameters that measures the change in fitness and performance of the taekwondo athletes which were carried out before and after enrolling in the program (Table 3) revealed improvement in the performance and muscle endurance. The anaerobic system for energy production in which lactic acid is liberated was assessed by two parameters; the muscle endurance and the performance endurance each for one min. The improvement in muscle endurance was measured by counting the number of each of setups and pushups per one minute and that for the performance endurance by the exercise of the front turning kick for one min for each participant before and after enrolling in the program. Results (Table 3) revealed a very highly significant increase in the number of setups and pushups and 
Table 3: Exercises for assessment of anaerobic power for muscle and performance endurance, motor speed and explosive strength before and after having the adaptogenic formula for all participants

\begin{tabular}{|c|c|c|c|c|}
\hline \multirow[b]{2}{*}{ Parameters } & \multicolumn{3}{|c|}{ Subjects $(n=15)$} & \multirow[b]{2}{*}{ p-value } \\
\hline & Before & After & Change (\%) & \\
\hline \multicolumn{5}{|l|}{ Muscle endurance for $1 \mathrm{~min}$} \\
\hline Setups for 1 min (No.) & $55 \pm 0.94$ & $68 \pm 0.73^{*}$ & 23.6 & 0.000 \\
\hline Pushups (No.) & $45 \pm 1.96$ & $57 \pm 1.95^{*}$ & 26.7 & 0.000 \\
\hline \multicolumn{5}{|l|}{ Motor speed for $15 \mathrm{sec}$} \\
\hline Right front turning kick (No.) & $25 \pm 1.69$ & $28 \pm 1.70^{*}$ & 12.0 & 0.000 \\
\hline Left front turning kick (No.) & $24 \pm 1.15$ & $27 \pm 0.96^{*}$ & 12.5 & 0.000 \\
\hline Right-left front turning kick (No.) & $13 \pm 0.71$ & $15 \pm 0.069$ & 15.4 & 0.204 \\
\hline \multicolumn{5}{|l|}{ Explosive strength } \\
\hline Standing long jumping $(\mathrm{cm})$ & $190 \pm 1.65$ & $225 \pm 1.68^{*}$ & 18.4 & 0.000 \\
\hline \multicolumn{5}{|l|}{ Performance endurance for $1 \mathrm{~min}$} \\
\hline Right front turning kick (No.) & $50 \pm 0.79$ & $62 \pm 0.93^{*}$ & 24.0 & 0.000 \\
\hline Left front turning kick (No.) & $49 \pm 0.55$ & $60 \pm 1.40^{*}$ & 22.4 & 0.000 \\
\hline Right-left front turning kick (no.) & $26 \pm 0.50$ & $32 \pm 0.66^{*}$ & 23.1 & 0.000 \\
\hline
\end{tabular}

Data are represented as Mean \pm SE. Values are significant at $p<0.05$, otherwise are not significant. No.: Number, ${ }^{*}$ Denotes significant

for the front turning kick for one min revealed a highly significant increase in the number of counts per one min either for the right alone or left alone or right and left together by a percent increase of $24,22.4$ and $23.1 \%$, respectively. The obtained results can be explained on the basis of lactic acid formation due to intensive exercise and its removal after adaptogen intake. Lactic acid is known to be liberated in serve exercise during the anaerobic oxidation to cover body's need and demand from energy by the rapid pathway of energy production which is the anaerobic system ${ }^{40}$. The more the clearance of the lactic acid, the more the recovery from muscle soreness and consequently the more the increase in the number of setups, pushups and the front turning kick each per one min. L-arginine supplementation was reported to facilitate the removal of lactic acid produced during intensive exercise and thus reduces fatigue and recovery time $e^{4,41}$. The other anaerobic system for rapid production of energy within few seconds is the ATP-PC system which liberates inorganic phosphate as a by-product whose accumulation causes fatigue ${ }^{42}$. Two parameters tested this system, the motor speed for $15 \mathrm{sec}$ and the explosive strength (Table 3). Motor speed was measured by front turning kick for $15 \mathrm{sec}$ and the results revealed a significant improvement in counts per $15 \mathrm{sec}$, either right alone or left alone or right and left together with a percent increase of $12,12.5$ and $15.4 \%$, respectively. Results for the standing long jumping, which measured the explosive strength, revealed an improvement evidenced by increasing the jump by $18 \%$ after having the adaptogenic formula compared to the baseline. It was reported that there was an increase in anaerobic power by L-arginine supplementation ${ }^{15,43}$.
Biochemical parameters: Biochemical parameters that reflect the clearance of the accumulated by-products of the anaerobic oxidation resulted from the intensive exercise, so as to prevent muscle soreness and consequently enhance the performance, such as serum lactate dehydrogenase-5 (LDH-5), creatine kinase (CK), cortisol and nitric oxide (NO) were determined (Fig. 1a-d). The LDH-5 is responsible for the clearance of lactate which is the by-product of anaerobic lactic oxidation while CK for clearance of inorganic phosphate which is the by-product of the phosphogen system. Muscle cell degradation releases these two enzymes into the circulation. Their presence is indicative of muscular damage ${ }^{21}$.

Activity of Lactate dehydrogenase-5 (LDH-5): The value of serum LDH-5 activity (Fig. 1a) amounted to 171.64 $\pm 9.44 \mathrm{UL}^{-1}$ for subjects before being enrolled in the program and decreased to a value of $113.08 \pm 15.69 \mathrm{UL}^{-1}$ when analyzed at the end of the program with a percentage change of $-33.91 \%$. This is an indication that the adaptogen given to the subjects lowered the activity of the circulating $\mathrm{LDH}-5$. It was previously mentioned that lactate accumulation in muscle cells leads to acidosis and muscular damage which in turn causes fatigue ${ }^{44}$. Results obtained by Kim et al. ${ }^{12}$, who studied the effect of acute red ginseng intake on male volunteers for recovery from repeated anaerobic exercise, seemed to be in accordance with our results. Panax ginseng, one of the ingredients used to compose the adaptogen in the present study was reported previously to combat fatigue and enhance physical performance ${ }^{38}$. It was also reported to have a vasodilator effect ${ }^{45}$, to enhance the blood circulation and to lower the arterial stiffening ${ }^{46}$. In addition, the strong antioxidant activity 

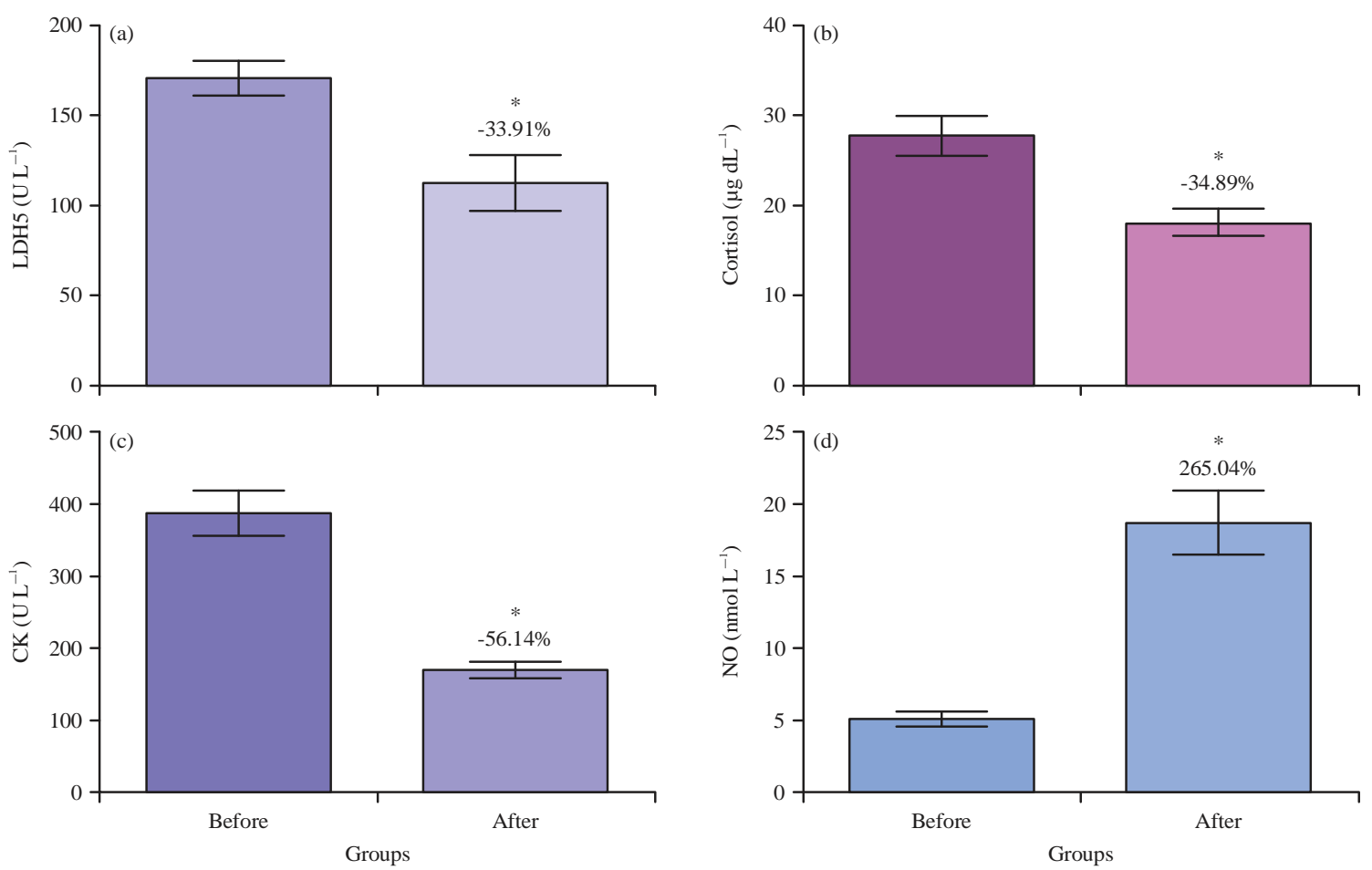

Fig. 1(a-d): Parameters of catabolic status, (a) Lactate dehydrogenase isoenzyme- 5 (LDH5) activity $p<0.004$, percentage change is $-33.91 \%$, (b) Cortisol concentration, $p<0.000$, percentage change is $-34.89 \%$, (c) Creatine kinase activity (CK) $p<0.000$, percentage change is $-56.14 \%$ and (d) Nitric oxide concentration (NO) $p<0.001$, percentage change is $265.04 \%$ Bars represent Mean \pm SE. Values are significant at $p<0.05$, otherwise are not significant. Significant values are denoted by*

of the ginsenosides ${ }^{13}$, the active components of red ginseng, may act to prevent the oxidation of the cellular membrane of the muscle cells and prevents the leakage of muscular LDH-5 into the circulation. Also, Gupta et al. ${ }^{47}$ suggested that supplementation of L-arginine acts at a cellular level to maintain the energy-dependent process of membrane permeability and in turn prevent leakage of muscular LDH-5 enzyme into the circulation, consequently lowering its activity in the circulation. Chen et $a / .^{48}$ reported that whey protein supplementation decreased the level of LDH activity in the circulation of trained mice.

Concentration of cortisol: Concentration of serum cortisol was significantly decreased when subjects were enrolled in the study and given the adaptogen (Fig. 1b). The values obtained were $27.77 \pm 2.23 \mu \mathrm{g} \mathrm{dL}{ }^{-1}$ before enrolling in the program which is considered as a high value (the known normal range is up to $13.51 \mu \mathrm{g} \mathrm{dL}^{-1}$ in male). This value became $18.08 \pm 1.47 \mu \mathrm{g} \mathrm{dL}^{-1}$ after entering the program which is nearly normal. It is worth mentioning that Hill et al.22 reported an increase in circulating cortisol level as a response to the physical exercise. Once released in the circulation, cortisol is up-taken by different tissues such as skeletal muscles, liver and adipose tissue where it mediates catabolic physiological processes that helps to increase exercise capacity. In skeletal muscles, it promotes proteins to be broken down into amino acids while stimulating gluconeogenesis in liver to provide additional energy ${ }^{49,50}$. Ginseng was reported to treat patients having disorders related to the hypersecretion of cortisol ${ }^{51}$.

Activity of creatine kinase (CK): A remarkable finding is that the plasma creatine kinase (CK), the enzyme that catalyzes the creatine conversion into phosphocreatine (PC) utilizing adenosine triphosphate (ATP) ${ }^{52}$, significantly dropped from $387.41 \pm 31.04 \mathrm{U} \mathrm{L}^{-1}$ at baseline to $169.91 \pm 11.60 \mathrm{U} \mathrm{L}^{-1}$ after 4 weeks of enrolling in the program (Fig. 1C). The CK levels were reported to be elevated following heavy exercise and it may be increased up to 30 folds the normal range within $24 \mathrm{~h}$ of extensive physical activity and declines slowly within 7 days ${ }^{53}$. This enzyme usually exists in the muscle tissue and its occurrence in serum indicates muscle damage or increased cell permeability due to intensive exercise. Jung et al.54 reported similar results of increasing the circulating CK due to severe exercise that induce muscle damage after uphill treadmill running in humans and supplementation of Panax ginseng resulted in lowering creatine kinase activity. Reduction of the activity of CK in the circulation as occurred in 
the present study is indicative of the ability of the formula to minimize liberation of this enzyme in the circulation. A similar finding was reported by Gupta et al. ${ }^{47}$, who mentioned that L-arginine counteracted the increased cell membrane permeability and cellular membrane damage on exposure to stressful conditions as it stabilizes the cell membrane due to its antioxidant potency thus prevent the leakage of the enzyme creatine kinase into the circulation, hence decreased its activity.

Concentration of nitric oxide (NO): Another finding is that the level of plasma NO of subjects enrolled in the present study increased significantly, the value obtained after giving the adaptogen was more than 3 times the value before with a percent change of $265.04 \%$ (Fig. 1d). Similar results were reported before by Lomonosova et $\mathrm{a} / \mathrm{K}^{39}$, who mentioned that supplementation of L-arginine which is a precursor of nitric oxide prior to single bout of eccentric exercise in rats led to alleviation of muscle fiber damage through increasing NO content of muscle fibers. Civan et al. ${ }^{55}$ reported similar results of increased NO by ginseng supplementation in women athletes. This was explained depending on vascular effect of ginseng which is mediated by pronounced increase in the expression of endothelial nitric oxide synthase (eNOS) and thus a rise in the production of nitric oxide ${ }^{45}$ which increases blood flow and oxygen to the skeletal muscle ${ }^{56}$ and thus helps for clearance of the accumulated by-products resulted from extensive exercise.

\section{Concentration of malondialdehyde and total antioxidant} capacity: Regarding the state of oxidation and generation of free radicals in the body, there was a decreased generation of free radicals as indicated by a slight non-significant decrease in serum malondialdehyde level which is a measure of the oxidation product in the body after enrolling in the program (Fig. 2a, b). Also, the value of plasma total antioxidant capacity (TAC) recorded a slightly non-significant increase after the end of the program. Such pattern shows that the body during the exercise was not subjected to drastic oxidation and formation of free radicals because the antioxidants present in the constituents of the formula particularly those of ginseng could exert their action and protect against the oxidative stress. Panaxginseng extracts have been reported to up regulate the antioxidant defense system of the body as well as attenuating the oxidative stress in rats ${ }^{57}$. Also, ginsenosides, the main bioactive components of ginseng were reported to have a potent free radical scavenging activity ${ }^{58,46}$. Kim et al. ${ }^{59}$ studied the effect of supplementation with Panax ginseng extract on healthy male subjects that performed exhaustive exercise and they reported a decrease in the level of plasma MDA and an
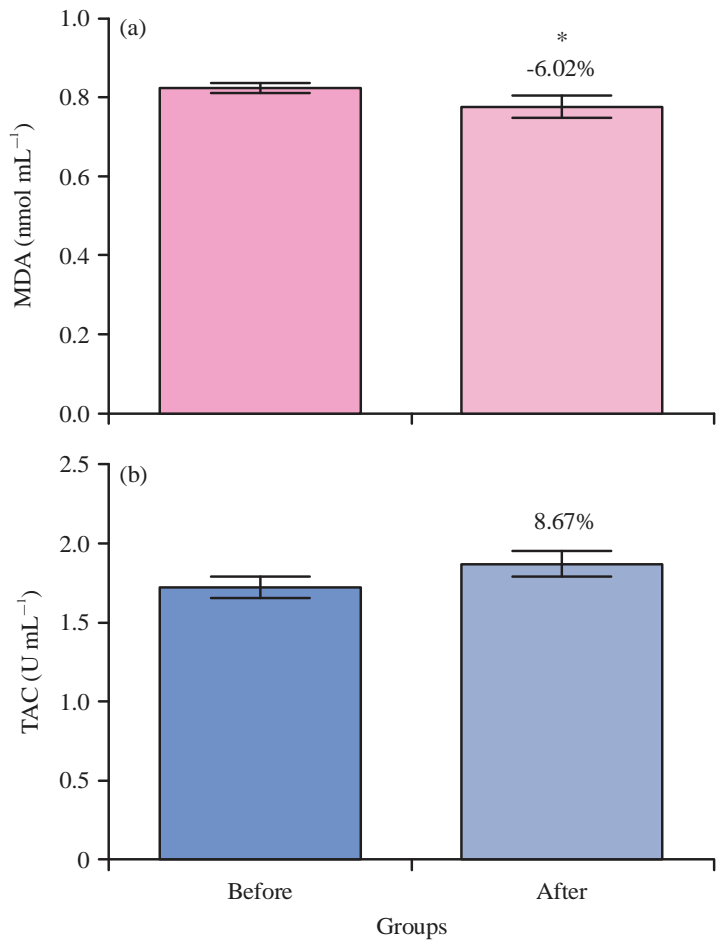

Fig. 2(a-b): Parameters of antioxidant status: (a) Lipid peroxidation product concentration; malondialdehyde (MDA) $p<0.678$, percentage change is $-6.02 \%$ and (b) total antioxidant capacity (TAC) $p<0.07$, percentage change is $8.67 \%$

Bars represent Mean \pm SE. Values are significant at $p<0.05$, otherwise are not significant

increase in the activity of both superoxide dismutase and catalase and they attributed their obtained results to the antioxidant effect of ginseng extract. Also, whey protein was reported to decrease MDA and increase TAC when given to rats with liver toxicity due to its antioxidant activity as it increased cellular reduced glutathione (GSH) level since it contains cysteine which is the building block for glutathione (GSH) synthesis ${ }^{60,61}$. In addition, Gupta et al. ${ }^{47}$ reported a decrease in MDA and an increase in the activity of antioxidant enzymes by L-arginine supplementation in rats subjected to generalized and comprehensive stress.

\section{Liver function, kidney function and complete blood count}

(CBC): Regarding the general safety of the formula, Table 4 and 5 show the values of the parameters reflecting the state of the liver such as AST, ALT and total protein and the kidney function represented by the values of urea and creatinine and complete blood count (CBC). As shown, there were no statistically significant differences between the values obtained for these parameters before and after admission in 
Table 4: Liver function and kidney function of the studied subjects

\begin{tabular}{|c|c|c|c|c|}
\hline \multirow[b]{2}{*}{ Parameters } & \multicolumn{3}{|c|}{ Subjects $(n=15)$} & \multirow[b]{2}{*}{$p$-value } \\
\hline & Before & After & Change (\%) & \\
\hline$\overline{\mathrm{AST}}\left(\mathrm{U} \mathrm{L}^{-1}\right)$ & $10.50 \pm 0.70$ & $8.33 \pm 0.52^{*}$ & -20.67 & 0.050 \\
\hline $\operatorname{ALT}\left(U L^{-1}\right)$ & $10.99 \pm 0.28$ & $10.59 \pm 0.66$ & -3.64 & 0.590 \\
\hline Total protein $\left(\mathrm{g} \mathrm{dL}^{-1}\right)$ & $7.05 \pm 0.35$ & $7.38 \pm 0.39$ & 4.68 & 0.070 \\
\hline Urea $\left(\mathrm{mg} \mathrm{dL}^{-1}\right)$ & $37.27 \pm 1.87$ & $34.57 \pm 1.72$ & -7.24 & 0.189 \\
\hline Creatinine $\left(\mathrm{mg} \mathrm{dL}^{-1}\right)$ & $1.23 \pm 0.06$ & $1.10 \pm 0.04$ & -10.57 & 0.860 \\
\hline \multicolumn{5}{|c|}{ Data are represented as Mean \pm SE. Values are significant at $p<0.05$, otherwise are not significant, ${ }^{*}$ Denotes significant } \\
\hline \multicolumn{5}{|c|}{ Table 5: Complete blood count (CBC) of the studied subjects } \\
\hline & \multicolumn{3}{|c|}{ Subjects $(\mathrm{n}=15)$} & \multirow[b]{2}{*}{$p$-value } \\
\hline Parameters & Before & After & Change (\%) & \\
\hline $\mathrm{Hb}\left(\mathrm{g} \mathrm{dL}^{-1}\right)$ & $15.37 \pm 0.45$ & $15.29 \pm 0.39$ & -0.52 & 0.622 \\
\hline $\operatorname{RBCs}\left(10^{6} \mu \mathrm{L}^{-1}\right)$ & $5.18 \pm 0.15$ & $5.23 \pm 0.14$ & 0.97 & 0.466 \\
\hline WBCs $\left(10^{3} \mu \mathrm{L}^{-1}\right)$ & $6.66 \pm 0.60$ & $6.63 \pm 0.40$ & -0.03 & 0.939 \\
\hline HCT (\%) & $42.39 \pm 0.13$ & $44.01 \pm 0.91^{*}$ & 3.82 & 0.005 \\
\hline MCV (fL) & $82.04 \pm 1.56$ & $84.29 \pm 1.47^{*}$ & 2.74 & 0.000 \\
\hline $\operatorname{PLT}\left(10^{3} \mu \mathrm{L}^{-1}\right)$ & $240.86 \pm 0.35$ & $322.88 \pm 16.89^{*}$ & 34.05 & 0.000 \\
\hline
\end{tabular}

Data are represented as Mean \pm SE. Values are significant at $p<0.05$, otherwise are not significant. Hb: Hemoglobin concentration, RBCs: Red blood corpuscles, WBCs: White blood cells, HCT: Hematocrit value, MCV: Mean corpuscular volume, PLT: Platelet count, *Denotes significant

the program for 4 weeks except for a slight but significant drop in the activity of plasma AST. This decline may be regarded as a good sign for improving the performance and recovery from fatigue, since AST was previously described as one of the muscle enzymes ${ }^{53}$ and was reported before to be one of the biochemical parameters reflecting the post-workout fatigue ${ }^{62}$. Another biochemical parameter that evaluates the safety of the prepared formula is the complete blood count (Table 5). Also, there was no change of clinical significance in any of the analyzed parameters for CBC, except for a significant increase in the platelet count. This increase may be due to the potentiating effect of ginseng on bone marrow, particularly on the platelets which was mentioned previously by Raghavendran et al. ${ }^{63}$, who reported that Korean red ginseng enhances myelopoiesis as it promotes platelet count after myelosuppression induced by 5 -fluorouracil in mice. In fact, most of the ingredients of the formula were reported to be safe in many previous studies. Lee and Rhee. ${ }^{51}$ mentioned that ginseng supplements are safe, whey protein was reported to be safe for most adults when being used appropriately ${ }^{64}$ and L-arginine was also reported to be safe and well tolerated up to $20 \mathrm{~g} /$ day in studies with healthy subjects $^{65,15}$.

\section{CONCLUSION}

Based on the obtained results, it can be concluded that the constituents of the composed adaptogenic formula act in a synergistic way and reinforce each other without any health hazards so that it can be used with no restriction and for longer time. Using this formula by athletes of anaerobic exercises leads to getting rid of anaerobic by-products that helps to combat muscular fatigue, enhance recovery time thus improve athletic performance and anaerobic endurance.

\section{SIGNIFICANCE STATEMENT}

This study discovered that providing athletes of anaerobic exercises with the newly composed adaptogenic formula promoted their anaerobic power as it succeeded to combat fatigue and reduce the recovery time by removing the undesirable by-products of the anaerobic exercises. Thus, it was able to enhance the physical performance and improve the anaerobic endurance. This will help athletes to have the chance for participating in much more international competitions and for playing with their most optimum performance so as to achieve their ultimate aim of having new high records in their sports. Also, this study will help and enable the researchers for better understanding of the biochemical changes accompanying the high-intensity exercises and how we can restore these changes to overcome fatigue and perform better.

\section{ACKNOWLEDGMENTS}

The present study is a part of a project approved and granted by the National Research Centre in Egypt (2013-2016) and entitled "Production of Dietary Supplements to Compete with Imported Items.", project number"10090005". All authors would like to acknowledge National Research Centre for funding this study and for providing laboratory facilities for the research study. 


\section{REFERENCES}

1. Melhim, A.F., 2001. Aerobic and anaerobic power responses to the practice of taekwon-do. Br. J. Sports Med., 35: 231-234.

2. Cho, K.O., C.E. Garber, S. Lee and Y.S. Kim, 2013. Energy balance during Taekwondo practice in elite male Taekwondo players. J. Lifestyle Med., 3: 54-61.

3. Cases, J., C. Romain, C. Marin-Pagan, L.H. Chung and J.M. Rubio-Perez et al., 2017. Supplementation with a polyphenol-rich extract, PerfLoad ${ }^{\circledR}$, improves physical performance during high-intensity exercise: A randomized, double blind, crossover trial. Nutrients, Vol. 9. 10.3390/nu9040421.

4. Westerblad, H., D.G. Allen and J. Lannergren, 2002. Muscle fatigue: Lactic acid or inorganic phosphate the major cause? Physiology, 17: 17-21.

5. De Souza, C.F. and L.C. Fernandes, 2006. Production of reactive oxygen species during the aerobic and anaerobic exercise. Brazil. J. Kinanthropomet. Hum. Perfor., 8: 102-109.

6. Ishii, H. and Y. Nishida, 2013. Effect of lactate accumulation during exercise-induced muscle fatigue on the sensorimotor cortex. J. Phys. Ther. Sci., 25: 1637-1642.

7. Baker, J.S., M.C. McCormick and R.A. Robergs, 2010. Interaction among skeletal muscle metabolic energy systems during intense exercise. J. Nutr. Metab., Vol. 2010. 10.1155/2010/905612.

8. Panossian, A. and G. Wikman, 2010. Effects of adaptogens on the central nervous system and the molecular mechanisms associated with their stress-protective activity. Pharmaceuticals, 3: 188-224.

9. Panossian, A., 2017. Understanding adaptogenic activity: Specificity of the pharmacological action of adaptogens and other phytochemicals. Ann. NY Acad. Sci., 140: 49-64.

10. Hsu, C.C., M.C. Ho, L.C. Lin, B. Su and M.C. Hsu, 2006. American ginseng supplementation attenuates creatine kinase level induced by submaximal exercise in human beings. World J. Gastroenterol., 11: 5327-5331.

11. Lee, N.H., H.C. Jung and S. Lee, 2016. Red ginseng as an ergogenic aid: A systematic review of clinical trials. J. Exerc. Nutr. Biochem., 20: 13-19.

12. Kim, S., J. Kim, Y. Lee, M.K. Seo and D.J. Sung, 2016. Anti-fatigue effects of acute red ginseng intake in recovery from repetitive anaerobic exercise. Iran. J. Public Health, 45: 387-389.

13. Choi, K.T., 2008. Botanical characteristics, pharmacological effects and medicinal components of Korean Panax ginseng C A Meyer. Acta Pharmacol. Sinica, 29: 1109-1118.

14. Yu, S.H., H.Y. Huang, M. Korivi, M.F. Hsu and C.Y. Huang et al., 2012. Oral Rg1 supplementation strengthens antioxidant defense system against exercise-induced oxidative stress in rat skeletal muscles. J. Int. Soc. Sports Nutr., Vol. 9. 10.1186/1550-2783-9-23.
15. Alvares, T.S., C.M. Meirelles, Y.N. Bhambhani, V.M. Paschoalin and P.S. Gomes, 2011. L-Arginine as a potential ergogenic aidin healthy subjects. Sports Med., 41: 233-248.

16. Bailey, S.J., P.G. Winyard, A. Vanhatalo, J.R. Blackwell, F.J. DiMenna, D.P. Wilkerson and A.M. Jones, 2010. Acute l-arginine supplementation reduces the $\mathrm{O}_{2}$ cost of moderate-intensity exercise and enhances high-intensity exercise tolerance. J. Applied Physiol., 109: 1394-1403.

17. Williams, M., 2005. Dietary supplements and sports performance: Amino acids. J. Int. Soci. Sports Nutr., 2: 63-67.

18. Kerksick, C.M., C.J. Rasmussen, S.L. Lancaster, B. Magu and P. Smith et al., 2006. The effects of protein and amino acid supplementation on performance and training adaptations during ten weeks of resistance training. J. Strength Cond. Res., 20: 643-653.

19. Flaim, C., M. Kob, A.M. Di Pierro, M. Herrmann and L. Lucchin, 2017. Effects of a whey protein supplementation on oxidative stress, body composition and glucose metabolism among overweight people affected by diabetes mellitus or impaired fasting glucose: A pilot study. J. Nutr. Biochem., 50: 95-102.

20. Hamarsland, H., A.L. Nordengen, S.N. Aas, K. Holte and I. Garthe et al., 2017. Native whey protein with high levels of leucine results in similar post-exercise muscular anabolic responses as regular whey protein: A randomized controlled trial. J. Int. Soc. Sports Nutr., Vol. 14. 10.1186/s12970-017-0202-y.

21. Nie, J., T.K. Tong, K. George, F.H. Fu, H. Lin and Q. Shi, 2011. Resting and post-exercise serum biomarkers of cardiac and skeletal muscle damage in adolescent runners. Scand. J. Med. Sci. Sports, 21: 625-629.

22. Hill, E.E., E. Zack, C. Battaglini, M. Viru, A. Viru and A.C. Hackney, 2008. Exercise and circulating cortisol levels: The intensity threshold effect. J. Endocrinol. Investigat., 31: 587-591.

23. AOAC., 1995. Official Methods of Analysis: Sec. 33.2.11, Method 991.20. 16th Edn., AOAC International, Gaithersburg, MD., USA.

24. AOAC., 2012. Official Methods of Analysis: No. 994.12. 19th Edn., AOAC international, USA., pp: 18-19.

25. Spungen, A.M., W.A. Bauman, J. Wang and R.N. Pierson, 1995. Measurement of body fat in individuals with tetraplegia: A comparison of eight clinical methods. Spinal Cord, 33: 402-408.

26. Kenett, D., 1988. Quantitative ELISA for human lactate dehydrogenase isoenzyme 5. J. Immunoassay, 9: 37-49.

27. Watts, N.B. and G.T. Tindall, 1988. Rapid assessment of corticotropin reserve after pituitary surgery. JAMA, 259: 708-712.

28. Gerhardt, W. and J. Waldenstrom, 1979. Creatine kinase B-subunit activity in serum after immunoinhibition of M-subunit activity. Clin. Chem., 25: 1274-1280. 
29. Montgomery, H.A.C. and J.E. Dymock, 1961. Determination of nitrite in water. Analyst, 86: 414-416.

30. Draper, H.H. and M. Hadley, 1990. Malondialdehyde determination as index of lipid peroxidation. Meth. Enzymol., 186: 421-431.

31. Koracevic, D., G. Koracevic, V. Djordjevic, S. Andrejevic and V. Cosic, 2001. Method for the measurement of antioxidant activity in human fluids. J. Clin. Pathol., 54: 356-361.

32. Henry, R.J., D.C. Chiamori, O. Golub and S. Berkman, 1960. Revised spectrophotometric methods for the determination of glutamic-oxalacetic transaminase, glutamic-pyruvic transaminase and lactic acid dehydrogenase. Am. J. Clin. Pathol., 34: 381-398.

33. Koller, A., 1984. Total Serum Protein. In: Clinical Chemistry: Theory, Analysis and Correlation, Kaplan, L.A. and A.J. Pesce (Eds.). Mosby Company, St. Louis, MO., USA., ISBN-13: 978-0801627057, pp: 418, 1316-1324.

34. Fawcett, J.K. and J.E. Scott, 1960. A rapid and precise method for the determination of urea. J. Clin. Pathol., 13: 156-159.

35. Murray, R.L., 1984. Creatinine. In: Clinical Chemistry, Theory, Analysis and Correlation, Kaplan, L.A. and A.J. Pesce (Eds.)., CV Mosby Co., St. Louis, Toronto, Princeton, pp: 1247-1253.

36. Chen, I.F., H.J. Wu, C.Y. Chen, K.M. Chou and C.K. Chang, 2016. Branched-chain amino acids, arginine, citrulline alleviate central fatigue after 3 simulated matches in taekwondo athletes: A randomized controlled trial. J. Int. Soc. Sports Nutr., Vol. 13. 10.1186/s12970-016-0140-0

37. Kennedy, D.O., J.L. Reay and A.B. Scholey, 2007. Effects of 8 weeks administration of Korean Panax ginseng extract on the mood and cognitive performance of healthy individuals. J. Ginseng Res., 31: 34-43.

38. Kim, H.G., J.H. Cho, S.R. Yoo, J.S. Lee and J.M. Han et al., 2013. Antifatigue effects of Panax ginseng C.A. Meyer: A randomized double-blind, placebo-controlled trial.PLos One, Vol. 8. 10.1371/journal.pone.0061271.

39. Lomonosova, Y.N., B.S. Shenkman, G.R. Kalamkarov, T.Y. Kostrominova and T.L. Nemirovskaya, 2014. L-arginine supplementation protects exercise performance and structural integrity of muscle fibers after a single bout of eccentric exercise in rats. Plos One, Vol. 9. 10.1371/journal.pone.0094448.

40. Scott, C.B., 2005. Contribution of anaerobic energy expenditure to whole body thermogenesis. Nutr. Metab., Vol. 2. 10.1186/1743-7075-2-14

41. Schaefer, A., F. Piquard, B. Geny, S. Doutreleau and E. Lampert et al., 2002. L-arginine reduces exercise-induced increase in plasma lactate and ammonia. Int. J. Sports Med., 23: 403-407.

42. Takeuchi, T., M. Kushida, M. Kitayama, A. Fujita and F. Hata, 2004. Origin of ATP for $\mathrm{Ca}^{2+}$-induced contraction in the guinea-pig femoral artery. Naunyn-Schmiedeberg's Arch. Pharmacol., 369: 350-357.
43. Chen, S., W. Kim, S.M. Henning, C.L. Carpenter and Z. Li, 2010. Arginine and antioxidant supplement on performance in elderly male cyclists: A randomized controlled trial. J. Int. Soc. Sports Nutr., Vol. 7. 10.1186/1550-2783-7-13.

44. Robergs, R.A., F. Ghiasvand and D. Parker, 2004. Biochemistry of exercise-induced metabolic acidosis. Am. J. Physiol.-Regulat. Integrat. Comparat. Physiol., 287: R502-R516.

45. Gillis, C.N., 1997. Panax ginseng pharmacology: A nitric oxide link. Biochem. Pharmacol., 54: 1-18.

46. Chrysant, S.G., G.S. Chrysant, 2017. Herbs used for the treatment of hypertension and their mechanism of action. Curr. Hypertens Rep., Vol. 19. 10.1007/s11906-017-0775-5.

47. Gupta, V., A. Gupta, S. Saggu, H.M. Divekar, S.K. Grover and R. Kumar, 2005. Anti-stress and adaptogenic activity of Farginine supplementation. Evidence-Based Complement. Altern. Med., 2: 93-97.

48. Chen, W.C., W.C. Huang, C.C.Chiu, Y.K. Chang and C.C. Huang, 2014. Whey protein improves exercise performance and biochemical profiles in trained mice. Med. Sci. Sports Exerc., 46: 1517-1524.

49. Viru, A. and M. Viru, 2004. Cortisol-essential adaptation hormone in exercise. Int. J. Sports Med., 25: 461-464.

50. Hackney, A.C., 2006. Stress and the neuroendocrine system: The role of exercise as a stressor and modifier of stress. Expert Rev. Endocrinol. Metab., 1: 783-792.

51. Lee, S. and D.K. Rhee, 2017. Effects of ginseng on stress-related depression, anxiety and the hypothalamic-pituitary-adrenal axis. J. Ginseng Res., 41: 589-594.

52. Wallimann, T., M. Wyss, D. Brdiczka, K. Nicolay and H.M. Eppenberger, 1992. Intracellular compartmentation, structure and function of creatine kinase isoenzymes in tissues with high and fluctuating energy demands: The 'phosphocreatine circuit' for cellular energy homeostasis. Biochem. J., 281: 21-40.

53. Moghadam-Kia, S., C.V. Oddis and R. Aggarwal, 2016. Approach to asymptomatic creatine kinase elevation. Cleveland Clin. J. Med.., 83: 37-42.

54. Jung, H.L., H.E. Kwak, S.S. Kim, Y.C. Kim, C.D. Lee, H.K. Byurn and H.Y. Kang, 2011. Effects of Panax ginseng supplementation on muscle damage and inflammation after uphill treadmill running in humans. Am. J. Chinese Med., 39: 441-450.

55. Civan, A., T. Kececi and E. Cakmakci, 2010. The effects of ginseng and exercise applications in sedentary individuals in women athletes on lipid hydroperoxide and nitric oxide. Ovidius Univ. Ann., Series Phys. Edu. Sport/Sci., Movement Health, 2: 774-777.

56. Clements, W.T., S.R. Lee and R.J. Bloomer, 2014. Nitrate ingestion: A review of the health and physical performance effects. Nutrients, 6: 5224-5264. 
57. Fu, Y. and L.L. Ji, 2003. Chronic ginseng consumption attenuates age-associated oxidative stress in rats. J. Nutr., 133: 3603-3603.

58. Lin, H.F., K. Tung, C.C. Chou, C.C. Lin, J. G. Lin and H. Tanaka, 2016. Panax ginsengand salvia miltiorrhiza supplementation abolishes eccentric exercise-induced vascular stiffening: A double-blind randomized control trial. BMC Complement. Altern. Med., Vol. 16. 10.1186/s12906-016-1139-4.

59. Kim, S.H., K.S. Park, M.J. Chang and J.H. Sung, 2005. Effects of Panax ginseng extract on exercise-induced oxidative stress. J. Sports Med. Phys. Fitness, 45: 178-182.

60. Gad, A.S., Y.A. Khadrawy, A.A. El-Nekeety, S.R. Mohamed, N.S. Hassan and M.A. Abdel-Wahhab, 2011. Antioxidant activity and hepatoprotective effects of whey protein and Spirulina in rats. Nutrition, 27: 582-589.

61. Forman, H.J., H. Zhang and A. Rinna, 2009. Glutathione: Overview of its protective roles, measurement and biosynthesis. Mol. Aspects Med., 30: 1-12.
62. Pettersson, J., U. Hindorf, P. Persson, T. Bengtsson, U. Malmqvist, V. Werkstrom and M. Ekelund, 2008. Muscular exercise can cause highly pathological liver function tests in healthy men. Br. J. Clin. Pharmacol., 65: 253-259.

63. Raghavendran, H.R.B., R. Sathyanath, J. Shin, H.K. Kim, J.M. Han, J. Cho and C.G. Son, 2012. Panax ginseng modulates cytokines in bone marrow toxicity and myelopoiesis: Ginsenoside Rg1 partially supports myelopoiesis. Plos One, Vol. 7. 10.1371/journal.pone.0033733.

64. Tsutsumi, R. and Y.M. Tsutsumi, 2014. Peptides and proteins in whey and their benefits for human health. Austin J. Nutri. Food Sci., Vol. 1.

65. Campbell, B., M. Roberts, C. Kerksick, C. Wilborn and B. Marcello et al., 2006. Pharmacokinetics, safety and effects on exercise performance of L-arginine $\alpha$-ketoglutarate in trained adult men. Nutrition, 22: 872-881. 